\title{
POLYNOMIAL APPROXIMATION OF FUNCTIONS ANALYTIC IN A DISK ${ }^{1}$
}

\section{J. T. SCHEICK}

Introduction. We consider the set $H$ of functions analytic in the open unit disk and put $\|f\|_{r}=\sup \{|f(z)|:|z|<r\}, 0<r \leqq 1$. Let $H_{M}=\left\{f \in H:\|f\|_{1} \leqq M\right\}, M>0 . P_{n}(n \geqq 0)$ is the set of polynomials of degree not exceeding $n$ while $P_{-1}=\{0\}$. The quantity

$$
E_{n}(f, r)=\inf \left\{\left\|f-p_{n}\right\|_{r}: p_{n} \in P_{n}\right\} \quad(0<r \leqq 1, n \geqq-1)
$$

is called the degree of approximation of $f$ by polynomials of degree not exceeding $n$ in $|z|<r$. For $A \subset H$,

$$
E_{n}(A, r)=\sup \left\{E_{n}(f, r): f \in A\right\}, \quad 0<r \leqq 1,
$$

is the degree of approximation of the class $A$. The object of this note is to determine $E_{n}(A, r)$ for many classes $A$. For the class $B^{(p)}=\left\{f \in H: f^{(p)} \in H_{1}\right\}, p=1,2,3 \cdots$ Babenko [1] has obtained

$$
E_{n-1}\left(B^{(p)}, r\right)=\frac{r^{n}}{n(n-1) \cdots(n-p+1)}, \quad n \geqq p, 0<r \leqq 1 .
$$

This result cannot be called completely unexpected, since there are earlier theorems in approximation theory which relate a bounded $p$ th derivative of $f$ to $E_{n}(f)$ by a formula similar to (1) (compare Favard's theorem).

We propose a new approach, based on Babenko's, yet simpler, and more general. It is close to some classical proofs, in particular to the proof of Favard's theorem in [2]. Babenko's theorem is extended to nonintegral $p$, another differential operator is considered, and many other classes yield up their degrees of approximation. The main theorem explains how to estimate $E_{n}(f, r)$ by a suitable factorization of the Taylor coefficients of $f$.

We will work with the product in $H$ :

$$
h * g(z)=\sum_{k=0}^{\infty} a_{k} b_{k} z^{k} \quad \text { where } \quad h(z)=\sum_{k=0}^{\infty} b_{k} z^{k}, \quad g(z)=\sum_{k=0}^{\infty} a_{k} z^{k},
$$

$$
h, g \in H \text {. }
$$

Then also $h * g \in H$. Put

Received by the editors February 14, 1966.

${ }^{1}$ This work has been supported in part by OSR, U. S. Air Force and Aerospace Research under contract no. AF 49(638)-1401. 


$$
A * g=\{f: f=h * g, h \in A\} .
$$

The aim is to compute the degree of approximation $E_{n-1}\left(H_{1} * g, r\right)$ for certain $g$. Since $E_{n-1}(f, r)=E_{n-1}\left(f+p_{n-1}, r\right)$ for each $p_{n-1} \in P_{n-1}$, we may assume $g(z)=\sum_{k=n}^{\infty} a_{k} z^{k}$ when convenient. The sequence $\left(a_{k}\right)_{k \geq n}$, where $n$ is a nonnegative in teger will be called positive when the following series converges and

(4) $\quad G(r, \theta)=\frac{1}{2} a_{n}+\sum_{k=1}^{\infty} a_{n+k} r^{k} \cos k \theta \geqq 0 \quad$ for $0 \leqq r<1, \theta$ real.

In this case, $g(z)=\sum_{k=n}^{\infty} a_{k} z^{k}$ belongs to $H$.

The main result.

THEOREM. Let $\left(a_{k}\right)_{k \geqq n}$ be positive and $f=g * h$ with $h(z)=\sum_{\mathbf{k}=0}^{\infty} b_{k} z^{k}$ $\in H_{1}$ and $g(z)=\sum_{k=n}^{\infty} a_{k} z^{k}$. Then, for each $0<R \leqq 1$, we have

$$
\begin{gathered}
\left\|f+p_{n-1}\right\|_{R} \leqq a_{n} R^{n}, \\
p_{n-1}(z)=p_{n-1}(R, z)=\sum_{k=0}^{n-1} b_{k} a_{2 n-k} R^{2}(n-k) z^{k} .
\end{gathered}
$$

Further, $f_{0}(z)=a_{n} z^{n}$ is extremal:

$$
f_{0} \in H_{1} * g \text { and } E_{n-1}\left(f_{0}, R\right)=a_{n} R^{n} .
$$

Hence

$$
E_{n-1}\left(H_{1} * g, R\right)=a_{n} R^{n} .
$$

For the proof we need the following

Leмma. Let $\left(A_{k}\right)_{k \geq n}$ be a positive sequence for which $\sum_{\boldsymbol{k}=n}^{\infty}\left|A_{k}\right|$ $<+\infty$. Then

$$
A_{n}=\min \left\{\frac{1}{2 \pi} \int_{0}^{2 \pi}\left|\sum_{k=-\infty}^{\infty} A_{k} e^{-i k \theta}\right| d \theta: \sum_{k=-\infty}^{n-1}\left|A_{k}\right|<+\infty\right\} .
$$

To prove this, put

$$
\Phi(\theta)=\sum_{k=-\infty}^{\infty} A_{k} e^{-i k \theta}
$$

Then

$$
\int_{0}^{2 \pi}|\Phi| d \theta \geqq\left|\int_{0}^{2 \pi} e^{i n \theta} \Phi(\theta) d \theta\right|=2 \pi A_{n}
$$

using the absolute convergence. On the other hand, selecting 
$A_{k}=A_{2 n-k}, k \leqq n-1$, we have $\sum_{k=-\infty}^{n-1}\left|A_{k}\right|<\infty$ and

$$
\begin{aligned}
\Phi_{0}(\theta) & =\sum_{k=-\infty}^{\infty} A_{k} e^{-i k \theta}=2 e^{-i n \theta}\left(\frac{1}{2} A_{n}+\sum_{k=1}^{\infty} A_{n+k} \cos k \theta\right) \\
& =2 e^{-i n \theta} G(\theta, 1)
\end{aligned}
$$

so that

$$
\int_{0}^{2 \pi}\left|\Phi_{0}\right| d \theta=2 \int_{0}^{2 \pi} G(\theta, 1) d \theta=2 \pi A_{n}
$$

Turning to the proof of the theorem, let $|z|=R<\rho<1$. We write the product (2) as an integral

$$
f(z)=h * g(z)=\sum_{k=n}^{\infty} a_{k} b_{k} z^{k}=\frac{1}{2 \pi i} \int_{|t|=\rho} h(t) g(z / t) t^{-1} d t .
$$

If $r=R / \rho$ and

$$
\sum_{k=-\infty}^{-1}\left|\xi_{k}\right| r^{|k|}<\infty
$$

then

$$
\begin{aligned}
f(z) & +\sum_{k=0}^{k-1} \xi_{k} b_{k} z^{k} \\
& =\frac{1}{2 \pi i} \int_{|t|=\rho} h(t)\left[\sum_{k=-\infty}^{-1} \xi_{k}\left(\overline{\frac{z}{t}}\right)^{-k}+\sum_{k=0}^{n-1} \xi_{k}\left(\frac{z}{t}\right)^{k}+\sum_{k=n}^{\infty} a_{k}\left(\frac{z}{t}\right)^{k}\right] \frac{d t}{t}
\end{aligned}
$$

due to the absolute convergence of the series (8) and the analyticity of $h$. Now letting $z / t=r e^{-i \theta}$, and taking absolute values, we obtain

$$
\begin{aligned}
\left|f(z)+\sum_{k=0}^{n-1} \xi_{k} b_{k} z^{k}\right| & \leqq \frac{i}{2 \pi} \int_{0}^{2 \pi}|\Phi| d \theta, \quad \Phi(\theta)=\sum_{k=-\infty}^{\infty} A_{k} e^{-i k \theta}, \\
A_{k} & =a_{k} r^{k}, k \geqq n, A_{k}=\xi_{k} r^{|k|}, k \leqq n-1 .
\end{aligned}
$$

Hence

$$
E_{n-1}(f, R) \leqq\left\|f+\sum_{k=0}^{n-1} \xi_{k} b_{k} u^{k}\right\|_{R} \leqq \frac{1}{2 \pi} \int_{0}^{2 \pi}|\Phi| d \theta
$$

$\left(u^{k}(z)=z^{k}\right)$ for all such $\Phi$. According to the lemma, we can minimize the right side by the choice $\xi_{k} r^{|k|}=a_{2 n-k} r^{2 n-k}, k \leqq n-1$, to obtain

$$
E_{n-1}(f, R) \leqq\left\|f+\sum_{k=0}^{n-1} a_{2 n-k} b_{k} r^{2(n-k)} u^{k}\right\|_{R} \leqq a_{n} r^{n}
$$


for each $\rho, R<\rho<1$. Now letting $\rho \rightarrow 1$, we get the first part of the theorem. Since $f_{0}=u^{n} * g, u^{n} \in H_{1}$, we have $f_{0} \in H_{1} * g$. From Rouché's theorem we conclude $\left\|f_{0}+p_{n-1}\right\|_{R} \leqq a_{n} R^{n}$ for all $p_{n-1} \in P_{n-1}$, so that $E_{n-1}\left(f_{0}, R\right)=a_{n} R^{n}$.

Applications. First some remarks on positive sequences $\alpha_{k}=a_{n+k}$, $k=0,1, \ldots$ It is known (Zygmund [3, Vol. 2, p. 150]) that $\alpha=\left(\alpha_{k}\right)$ is positive if and only if there exists an increasing function $F_{\alpha}$, defined and bounded on $[0,2 \pi]$ with

$\alpha_{k}=\frac{1}{\pi} \int_{0}^{2 \pi} \cos k \theta d F_{\alpha}(\theta), \quad 0=\frac{1}{\pi} \int_{0}^{2 \pi} \sin k \theta d F_{\alpha}(\theta), \quad k=0,1, \cdots$.

It is customary to extend $F_{\alpha}$ to all of the reals by $F_{\alpha}(\theta+2 \pi)=F_{\alpha}(\theta)$ $+F_{\alpha}(2 \pi)-F_{\alpha}(0)$. Let us say $F_{\alpha}$ generates $\alpha$. When $\alpha$ and $\beta$ are positive, the sequences $c \alpha=\left(c \alpha_{k}\right)(c \geqq 0), \alpha+\beta=\left(\alpha_{k}+\beta_{k}\right), \alpha * \beta=\left(\alpha_{k} \beta_{k}\right)$ are again positive. In the latter case, $\alpha * \beta$ is generated by

$$
F_{\gamma}(\theta)=\frac{1}{\pi} \int_{0}^{2 \pi} F_{\alpha}(\theta-t) d F_{\beta}(t), \quad \gamma=\alpha * \beta .
$$

(See Zygmund [3, Vol. 1, p. 38].) For example, if $\left(a_{k}\right)_{k \geqq n}$ and $\left(b_{k}\right)_{\kappa \geqq n}$ are positive and $g(z) \sum_{k=n}^{\infty} a_{k} z^{k}, h(z)=\sum_{k=n}^{\infty} b_{k} z^{k}$, we have

$$
E_{n-1}\left(H_{1} * g * h, R\right)=a_{n} b_{n} R^{n}, \quad 0<R \leqq 1 .
$$

Simple conditions that $\alpha$ be positive are

(9) $\alpha_{k} \geqq 0, \Delta \alpha_{k}=\alpha_{k+1}-\alpha_{k} \leqq 0, \Delta^{2} \alpha_{k}=\Delta\left(\Delta \alpha_{k}\right) \geqq 0, k=0,1, \cdots$.

To see this, one applies partial summation twice to the series in (4) to obtain the inequality (4). Note that if $\alpha$ and $\beta$ satisfy (9) then so do $c \alpha(c \geqq 0), \alpha+\beta, \alpha * \beta$, and $\left(\alpha_{k+p}\right)_{k \geqq 0}(p \geqq 0)$. Examples of sequences satisfying (9) are moment sequences $\alpha_{k}=\int_{0}^{1} t^{k} d q(t)$ with $q$ increasing and bounded in $[0,1]$. We have immediately

Proposition 1. If $g(z)=\sum_{k=p}^{\infty} a_{k} z^{k}$ and $\left(a_{k+p}\right)_{k \geq 0}$ satisfies (9), then

$$
E_{n-1}\left(H_{1} * g, R\right)=a_{n} R^{n}, \quad 0<R \leqq 1, n \geqq p .
$$

Now we can state an extension of Babenko's theorem which is contained in (10). If $p$ is a nonnegative real number we may define the $p$ th derivative of $f(z)=\sum_{k=0}^{\infty} c_{k} z^{k}(f \in H)$ by the equation

$$
z^{p} f^{(p)}(z)=\sum_{k=0}^{\infty} \frac{\Gamma(k+1)}{\Gamma(k-p+1)} c_{k} z^{k}
$$

where $z^{p}$ is real for real $z$ and $1 / \Gamma(-j)=0$ if $j=0,1, \ldots$. Let 
$B^{(p)}=\left\{f \in H:\left|f^{(p)}(z)\right| \leqq 1\right.$ for $\left.|z|<1\right\}$. Then we have the

Corollary. For each $p \geqq 0$ and $n \geqq[p]$,

$$
E_{n-1}\left(B^{(p)}, R\right)=\frac{\Gamma(n-p+1)}{\Gamma(k+1)} R^{n}, \quad 0<R \leqq 1 .
$$

Proof. We have $f \in B^{(p)}$ if and only if $f(z)=\sum_{k=0}^{[p]-1} c_{k} z^{k}+h * g(z)$ with $h(z) b=z^{p} f^{(p)}(z) \in H_{1}$ and $g(z)=\sum_{k=[p]}^{\infty} a_{k} z^{k}$, where $a_{k}=[\Gamma(k-p+1)$ $\left.\cdot \Gamma(k+1)^{-1}\right]$. One verifies immediately that the sequence $\left(a_{k}\right)_{k \geqq p}$ satisfies (9).

We can give another interpretation of our main result by means of differential operators. Let $T$ be the operator $u D$, that is,

$$
T f(z)=z f^{\prime}(z)=\sum_{k=0}^{\infty} k c_{k} z^{k} \quad \text { if } f(z)=\sum_{k=0}^{\infty} c_{k} z^{k}
$$

If $\psi(w)$ is a function defined for $w=n, n+1, \ldots$, where $n$ is a nonnegative integer, and if

$$
\limsup _{k \rightarrow \infty}|\psi(k)|^{1 / k} \leqq 1,
$$

we define the differential operator $\psi(T)$ from $H$ into $H$ by

$$
\psi(T) f(z)=\sum_{k=n}^{\infty} \psi(k) c_{k} z^{k} \quad \text { if } f(z)=\sum_{k=0}^{\infty} c_{k} z^{k} .
$$

That this definition is reasonable can be seen from the following example. Let $\psi(w)$ be an entire function of order not exceeding one and of minimal type if the order is one. Then $\lim \sup _{k \rightarrow \infty}\left(\sum_{j=0}^{\infty}\left|d_{j}\right| k^{j}\right)^{1 / k} \leqq 1$, where $\psi(w)=\sum_{j=0}^{\infty} d_{j} w^{j}$. (See Hille [4, Vol. 2, p. 183].) It follows that

$$
\psi(T) f(z)=\sum_{k=0}^{\infty} \psi(k) c_{k} z^{k}=\sum_{k=0}^{\infty} \sum_{j=0}^{\infty} d_{j} k^{j} c_{k} z^{k}=\sum_{j=0}^{\infty} d_{j} T^{j} f(z),
$$

the change in the order of summation being possible by the absolute convergence of the double series. The series on the right is the definition of $\psi(T) f$ to be found in Hille [4, Vol. 2, p. 48]. Let $S_{\psi}=\left\{f \in H: \psi(T) f \in H_{1}\right\}$ where $\psi(T) f$ is defined by (15). Now we can state

Proposition 2. Let $\psi(w)$ be defined and nonzero for $w=n, n+1, \cdots$ and satisfy (14). Then if $(1 / \psi(k))_{k \geqq n}$ is a positive sequence, we have

$$
E_{n-1}\left(S_{\psi}, R\right)=R^{n} / \psi(n), \quad 0<R \leqq 1 .
$$


Proof. We have $S=H_{1} * g$, where $g(z)=\sum_{k=n}^{\infty} \psi(k)^{-1} z^{k}$, and (16) follows from (6).

As a special case of Proposition 2 we state

Proposition 3. Formula (16) holds if $\psi(x)$ is defined for $x \geqq n$, satisfies (14) and also

$$
\psi(x)>0, \psi^{\prime}(x) \geqq 0,2 \psi^{\prime}(x)^{2} \geqq \psi(x) \psi^{\prime \prime}(x), \quad x \geqq n .
$$

Proof. Condition (17) means that $1 / \psi(x)$ is positive, increasing, and convex in $x \geqq n$. Hence $a_{k}=1 / \psi(k), k \geqq n$, satisfies (9).

For example, if $\psi(x)=x^{p}(p \geqq 0)$ or $\psi(x)=\prod_{k=1}^{\infty}\left(1+x / r_{k}\right)$ where $r_{k}>0$ and $\sum_{k=1}^{\infty} r_{k}^{-1}<+\infty$, then $\psi(x)$ satisfies the conditions of Proposition 3 (see Hille [4, Vol. 2, p. 195]). So does $\psi(x)=\sum_{j=0}^{m} d_{j} x^{i}$, if the $d_{j}$ are real and $d_{m}>0$, for $x \geqq n$, when $n$ is sufficiently large.

\section{REFERENCES}

1. K. I. Babenko, On the best approximation of a class of analytic functions, Izv. Akad. Nauk SSSR Ser. Mat. 22 (1958), 631-640.

2. G. G. Lorentz, Approximation of functions, Holt, Rinehart and Winston, New York, 1966.

3. A. Zygmund, Trigonometric series, 2nd ed., Cambridge University Press, London, 1959.

4. E. Hille, Analytic function theory, Ginn, New York, 1962.

Syracuse UNiversity 